\title{
Effects of Jinjiang Granules on Immunologic Function of Im- munosuppressive Mice Model Induced by Cyclophosphamide
}

Ming-san $\mathrm{MIAO}^{1, \star}$, Li-hua $\mathrm{CAO}^{1}$ and Xue-xia $\mathrm{ZHANG}^{1}$

\author{
${ }^{1}$ Henan University of Traditional Chinese Medicine, Zhengzhou 450008, Henan \\ Province, China
}

${ }^{*}$ Corresponding author

Keywords: Jinjiang Granules, Cyclophosphamide, Immunosuppressive Mice.

\begin{abstract}
Objective: To explore the effects of jinjiang granules on immunologic function and to provide evidence for jinjiang granules as the application of clinical medicine in the initial stage of cold, through the observation of the effects of jinjiang granules on immunologic function of low immune mouse model .Method: Mice were randomly and evenly divided into the normal control group(given distilled water), high-dose, mid-dose and low-dose jinjiang groups andpositive control group. There were 10 mice every group, weighing the body weight of the mice every 3 days and administering them drugs intragastrically once a day, doses of which were adjusted according to the weight changes for 14 successive days. After 2 hours of the last administration, weigh the body weight, and then sacrifice the mice with cervical dislocation before taking murine organ index test, hemolysin and hemolytic plaque test, peripheral blood lymphocytes transformation test, peritoneal macrophage phagocytic chicken red blood cells test, natural killer cell (NK) activity assay test (MMT)in mice. Result: Immunosuppressive mice models were duplicated successfully. Compared with blank group, there were significant differences in various indexes of mice model. Compared with Immunosuppressive mice models , medication group could improve the murine organ index, the level of hemolysin and hemolytic plaque, the transformation ability of peripheral blood lymphocytes, the ability of peritoneal macrophage phagocytosing chicken red blood cells, the activity of natural killer cell. Conclusion: Jinjiang granules can improve the immunologic function of either normal mice or immunosuppressive mice model. As the application of clinical medicine in the early stage of cold, Jinjiang granules can prevent common cold and reduce its symptom.
\end{abstract}

\section{Introduction}

Common cold is clinical common disease and frequently occuring illness with an average annual incidence of $70 \% \sim 80 \%$.Its treatment of Western medicine focuses on antiviral, simultaneeously, expectant and antibacterial treatment. The course of disease was about one week, and most could be cured completely. But parts usually appears repeated attack accompanied by symptoms of lung-yang deficiency, such as aversion to cold and cold limbs, shortness of breath, weakness, fatigue, dilute sputum, salivation, pale tongue, thin white tongue coating and so on. It is a failure to insist to continual treatment, which affects learning, work, and cause unnecessary economic burden and mental pressure. If we prevent the disease from the very beginning in the early stage of cold, we would alleviate patients' suffering and some unnecessary economic loss. However, most present medicine is aiming at symptoms after the onset of the disease and there is a lack of medicine which is used for cold in the early stage. So it is indispensable to research and develop this medicine.

Previous research result shows that Jinjiang granules have health function that it 
can clear heat and detoxify materials, evacuate inflammation, relieve sore throat, prevent common cold, improve immunity. It can improve normal mice immunity[1] . This experiment use cyclophosphamide to induce mice immunosuppressive to study the effects ofjinjiang granules on immunologic function further .

\section{Experimental material}

\section{Drug and reagent}

Jin jiang granules, Henan FuSen Pharmaceutical Co., Ltd., batch number: 20130226; Lentinus Edodes Polysaccharide tablet, HubeiGuangRen Pharmaceutical Co., Ltd., batchnumber: 121211;Cyclophosphamide, Tonghua MaoXiang Pharmaceutical Co., Ltd., batch number: 120801;Normal saline, Zhengzhou YongHe Pharmaceutical Co., Ltd., batch number: 12042401;Phytohemagglutinin(PHA), Shanghai Yihua Technology Co., Ltd., batch number: 20101128; Wright's stain solution, Haerbin Yuanyuan Medical Instrument Co., Ltd., batch number:20120103;RPMI-1640 Cultue medium, Beijing Solarbio Science \&Technology Co., Ltd., batch number: 20130307; DMSO, Beijing Solarbio Science \&Technology Co., Ltd., batch number: D8372.

\section{Experiment instrument}

UV-2000 ultraviolet spectrophotometer, Shanghai TianMei Scientific Instruments Co., Ltd.; BIORAD-680 enzyme mark instrument 680Microplate Reader, Bio-Rad Laboratories; KDC-160HR high-speed freezing centrifuge.

\section{Experimental animals}

Kunming mice, half male and half female, body weight 18 22, provided by Hubei Experimental Animal Center, certification number of the batch mice: 42000400000195.

\section{Methods}

\section{Effects on hemolysin and hemolytic plaque of immunosuppressive mice model} induced by cyclophosphamide

Sixty KM mice, half male and female, were divided into six groups randomly : high-dose, mid-dose and low-dose jinjiang groups, Lentinus Edodes Polysaccharide group, model group, blank group, which were respectively gavaged with high-dose, mid-dose and low-dose jinjiang granules solution $(12 \mathrm{~g} / \mathrm{kg}, 6 \mathrm{~g} / \mathrm{kg}$ and $3 \mathrm{~g} / \mathrm{kg}$, $0.2 \mathrm{~mL} / 10 \mathrm{~g})$, Lentinus Edodes Polysaccharide suspension $(30 \mathrm{mg} / \mathrm{kg}, 0.2 \mathrm{~mL} / 10 \mathrm{~g}$ ) and the same volume of distilled water for 7 days . During the first three days, all mice except normal group were injected intraperitoneally with cyclophosphamide $(80 \mathrm{mg} / \mathrm{kg}, 0.2 \mathrm{ml} / 10 \mathrm{~g})$, which were weighed every 3 days, and whose volume of administration was adjusted according to the weight changes . All mice were injected intraperitoneally with $0.2 \mathrm{~mL}$ suspension of $5 \%$ chicken red blood cell and saline on the first day. After 2 hours of the 7th day administration, all mice were extracted eyeballs for blood that were centrifuged to separate the serum and then diluted it with saline(1:10) . $1 \mathrm{ml}$ diluted serum was mixed well with $5 \%$ chicken red blood cell . In addition, take 5\% chicken red blood cell without mice serum as tube of blank control , and mix them well. After storing mixture in $37^{\circ} \mathrm{C}$ incubator for 30 minutes , terminate the reaction in ice water and then centrifuge to collect the supernatant whose absorbance could be measured with 721 ultraviolet spectrophotometer under the wavelength of 540nm and adjusted zero by blank tube . After the mice were sacri- 
ficed with cervical dislocation, spleen and thymus were taken out . Remove excess adipose tissue attached to spleen, and sip up blood by filter paper to weigh them respectivly. Two mice spleens of every group were homogenized with normal saline and regulate concentration of spleen cell to $5 \times 106 / \mathrm{mL}$ with saline $.0 .5 \mathrm{ml}$ spleen suspension , 2\% chicken red blood cell and 1:10 serum of guinea pig were mixed well in tube . In addition, take the same volume of normal saline without complement as tube of blank control, and mix them well . After storing mixture in $37^{\circ} \mathrm{C}$ incubator for 1 hour , centrifuge to collect the supernatant whose absorbance could be measured with 721 ultraviolet spectrophotometer under the wavelength of $413 \mathrm{~nm}$ and adjusted zero by blank tube .

\section{Effects on the ability of peritoneal macrophage phagocytosis and organ index of immunosuppressive mice model induced by cyclophosphamide .}

Sixty KM mice, half male and female, were divided into six groups randomly : high-dose, mid-dose and low-dose jinjiang groups, Lentinus Edodes Polysaccharide group , model group, blank group, which were respectively gavaged with high-dose,mid-dose and low-dose jinjiang granules solution $(12 \mathrm{~g} / \mathrm{kg}, 6 \mathrm{~g} / \mathrm{kg}$ and $3 \mathrm{~g} / \mathrm{kg}, 0.2 \mathrm{~mL} / 10 \mathrm{~g}$ ) , Lentinus Edodes Polysaccharide suspension $(30 \mathrm{mg} / \mathrm{kg}$, $0.2 \mathrm{~mL} / 10 \mathrm{~g}$ ) and the same volume of distilled water for 7 days . During the first three days , all mice except normal group were injected intraperitoneally with cyclophosphamide $(80 \mathrm{mg} / \mathrm{kg}, 0.2 \mathrm{ml} / 10 \mathrm{~g})$, which were weighed every 3 days, and whose volume of administrationwas adjusted according to the weight changes. All mice were injected intraperitoneally with $0.5 \mathrm{~mL}$ suspension of $5 \%$ chicken red blood after 2hours of the last administration and then sacrificed after 4hours of injection with chicken red blood . Inject mice intraperitoneally with $2.5 \mathrm{ml}$ Han`s solution, knead its abdomen slightly, scissor intraperitoneal skin , scissor a small hole in peritoneal, aspirate $2 \mathrm{ml}$ peritoneal fluid from it to tube, and mix it well . Aspirate a little peritoneal fluid whose droplet size is about $1.5 * 2 \mathrm{~cm}$ on the glass slide that were put on porcelain enamel disk with wet gauze to incubate for 30 minutes at $37^{\circ} \mathrm{C}$. Using normal saline to wash attached cells out, stain it with Wright's stain solution, and then it was rinsed with sterile distilled water and dried for use . Observe the situation of intraperitoneal macrophage phagocytosing chicken red blood cells under the microscope, and calculate phagocytic percentage, phagocytic index. Meanwhile spleen and thymus were taken out and weighed respectivly to calculate organ index .

phagocytic percentage $=$ (the number of macrophage of 100 macrophage phagocytosing chicken red blood cells/100)*100\%

phagocytic index $=$ the total number of chicken red blood cells phagocytosed by 100 macrophage/100

\section{Effects on the transformation ability of peripheral blood lymphocytes of immu- nosuppressive mice model induced by cyclophosphamide .}

Sixty KM mice, half male and female, were divided into six groups randomly : high-dose, mid-dose and low-dose jinjiang groups, Lentinus Edodes Polysaccharide group, model group, blank group, which were respectively gavaged with high-dose, mid-dose and low-dose jinjiang granules solution $(12 \mathrm{~g} / \mathrm{kg}, 6 \mathrm{~g} / \mathrm{kg}$ and $3 \mathrm{~g} / \mathrm{kg}$, $0.2 \mathrm{~mL} / 10 \mathrm{~g})$, Lentinus Edodes Polysaccharide suspension $(30 \mathrm{mg} / \mathrm{kg}, 0.2 \mathrm{~mL} / 10 \mathrm{~g})$ and the same volume of distilled water once a day for successive 7 days .The mice were weighed every 3 days, and whose volume of administration was adjusted according to the weight changes . During the first three days, all mice except normal group were injected intraperitoneally with cyclophosphamide $(80 \mathrm{mg} / \mathrm{kg}, 0.2 \mathrm{ml} / 10 \mathrm{~g})$. 
All mice were injected intramuscularly with PHA $10 \mathrm{mg} / \mathrm{kg}$ in the morning on the first to the third day of administration . In addition, some mice were injected and gavaged the same volume of normal saline as a completely blank control group . After2 hours of the 7th day administration, mice were sheared tail for blood that were smeared and stained with Wright's stain solution to observe the transformation rate of peripheral blood lymphocytes in the oil lens.

\section{Effects on the natural killer cell (NK) activity of immunosuppressive mice model induced by cyclophosphamide.}

Sixty KM mice, half male and female, were divided into six groups randomly : high-dose, mid-dose and low-dose jinjiang groups, Lentinus Edodes Polysaccharide group, model group, blank group, which were respectively gavaged with high-dose, mid-dose and low-dose jinjianggranules solution $(12 \mathrm{~g} / \mathrm{kg}, 6 \mathrm{~g} / \mathrm{kg}$ and $3 \mathrm{~g} / \mathrm{kg}$, $0.2 \mathrm{~mL} / 10 \mathrm{~g})$, Lentinus Edodes Polysaccharide suspension $(30 \mathrm{mg} / \mathrm{kg}, 0.2 \mathrm{~mL} / 10 \mathrm{~g})$ and the same volume of distilled water once a day for successive 7 days .The mice were weighed every 3 days, and whose volume of administration was adjusted according to the weight changes . During the first three days, all mice except normal group were injected intraperitoneally with cyclophosphamide $(80 \mathrm{mg} / \mathrm{kg}, 0.2 \mathrm{ml} / 10 \mathrm{~g})$.After2 days of the 7th day administration, the mice were sacrificed with cervical dislocation before taken murine spleens out respectively under aseptic condition, and then put them on plate with proper aseptic Hank's solution to separate NK cells with NK cells of spleen tissues separating medium kit .

Data analysis uses medical statistical pack SPSS 17.0 for windows to processs data statistically . Measurement data uses average \pm standard deviation to represent . One-way anova is applied for comparision among groups . LSD is used for data of equal variance, while Games-Howell is used for data of heterogeneity of variance.

\section{Result}

\section{Effects on hemolysin, hemolytic plaque of immunosuppressive mice model in- duced by cyclophosphamide}

Table1. Effectson hemolysin, hemolytic plaque of immunosuppressive mice model induced by cyclophosphamide (OD, $\left.\left.\overline{\overline{\mathrm{X}}}\right|_{\mathrm{s}}\right)$

\begin{tabular}{lllll}
\hline group & $\mathrm{n}$ & $\begin{array}{c}\text { Dose } \\
(\mathrm{g} / \mathrm{kg})\end{array}$ & hemolysin & hemolytic plaque \\
\hline Blank group & 10 & - & $0.457 \pm 0.033^{* *}$ & $0.428 \pm 0.031$ \\
model group & 10 & - & $0.368 \pm 0.032$ & $0.299 \pm 0.057 * *$ \\
Lentinus Edodes Polysaccharide group & 10 & 0.03 & $0.434 \pm 0.024^{* *}$ & $0.455 \pm 0.038$ \\
high-dose jinjiang groups & 10 & 12 & $0.442 \pm 0.017 * *$ & $0.431 \pm 0.028^{* *}$ \\
mid-dose jinjiang groups & 10 & 6 & $0.412 \pm 0.012^{* *}$ & $0.382 \pm 0.022^{* *}$ \\
low-dose jinjiang groups & 10 & 3 & $0.397 \pm 0.011^{* *}$ & $0.334 \pm 0.013$ \\
\hline
\end{tabular}

Note: *: represent that compared with model group $P<0.05 ; * *$ : represent that compared with model group $P<0.01$.

As Table1 shows that compared with blank group, hemolysin, hemolytic plaque level in model group has improved significantly $(P<0.01)$, it manifests inducing immunosuppressive model is successful . Compared with model group, hemolysin level in Lentinus Edodes Polysaccharide group, high-dose, mid-dose and low-dose jinjiang groups improved significantly on average $(P<0.01)$ and among them, Lentinus Edodes Polysaccharide group is optimal .Compared with model group, hemolytic plaque level in Lentinus Edodes Polysaccharide group, high-dose and mid-dose jinjiang groups 
improved significantly on average $(P<0.01)$ and among them, Lentinus Edodes Polysaccharide group is optimal.

Effects on the ability of peritoneal macrophage phagocytosis of immunosuppressive mice model induced by cyclophosphamide

Table2. Effectson the ability of peritoneal macrophage phagocytosisofimmunosuppressive mice model induced by cyclophosphamide $\left(\left.\overline{\bar{x}}\right|_{ \pm s}\right)$

\begin{tabular}{lllll}
\hline Group & $\mathrm{n}$ & $\begin{array}{c}\text { Dose } \\
(\mathrm{g} / \mathrm{kg})\end{array}$ & $\begin{array}{c}\text { phagocytic per- } \\
\text { centage }(\%)\end{array}$ & $\begin{array}{l}\text { Phagocytic } \\
\text { dex }\end{array}$ \\
\hline Blank group & 10 & - & $56.0 \pm 3.1^{* *}$ & $1.01 \pm 0.03^{* *}$ \\
model group & 10 & - & $42.9 \pm 2.4$ & $0.87 \pm 0.04$ \\
Lentinus Edodes Polysaccharide group & 10 & 0.03 & $54.1 \pm 3.2^{* *}$ & $1.20 \pm 0.03^{* *}$ \\
high-dose jinjiang groups & 10 & 12 & $53.6 \pm 2.8^{* *}$ & $1.15 \pm 0.02^{* *}$ \\
mid-dose jinjiang groups & 10 & 6 & $51.2 \pm 1.9^{* *}$ & $1.07 \pm 0.03^{* *}$ \\
low-dose jinjiang groups & 10 & 3 & $46.4 \pm 2.0^{*}$ & $0.99 \pm 0.03^{* *}$ \\
\hline
\end{tabular}

Note: *: represent that compared with model group $P<0.05$;**:represent that compared with model group $P<0.01$.

As Table 2 shows that compared with blank group, phagocytic transformation, phagocytic index level in model group has improved significantly $(P<0.01)$, it manifests inducing immunosuppressive model is successful. Compared with model group , phagocytic percentage and phagocytic index in Lentinus Edodes Polysaccharide group , high-dose and mid-dose jinjiang groups improved significantly on average $(P<0.01)$ and among them, High-dose jinjiang groups group is optimal. Phagocytic percentage and phagocytic index in low-dose jinjiang groups improved obviously on average $(P<0.05)$.

\section{Effects on the organ index of immunosuppressive mice model induced by cyclo- phosphamide}

Table3. Effectson the organ index of immunosuppressive mice model induced by cyclophospha-

$$
\operatorname{mide}(\overline{\bar{x}} \pm s)
$$

\begin{tabular}{lllll}
\hline Group & $\mathrm{n}$ & $\begin{array}{l}\text { Dose } \\
(\mathrm{g} / \mathrm{kg})\end{array}$ & $\begin{array}{c}\text { Spleenindex } \\
(\mathrm{mg} / 10 \mathrm{~g})\end{array}$ & $\begin{array}{l}\text { thymus index } \\
(\mathrm{mg} / 10 \mathrm{~g})\end{array}$ \\
\hline Blank group & 10 & - & $21.7 \pm 1.1^{* *}$ & $18.0 \pm 1.5^{* *}$ \\
model group & 10 & - & $15.2 \pm 1.2$ & $11.0 \pm 1.3$ \\
Lentinus Edodes Polysaccharide group & 10 & 0.03 & $22.2 \pm 1.8^{* *}$ & $18.9 \pm 1.7^{* *}$ \\
high-dose jinjiang groups & 10 & 12 & $21.4 \pm 1.7^{* *}$ & $19.1 \pm 1.8^{* *}$ \\
mid-dose jinjiang groups & 10 & 6 & $19.6 \pm 1.2^{* *}$ & $16.4 \pm 1.7^{* *}$ \\
low-dose jinjiang groups & 10 & 3 & $16.8 \pm 1.3^{*}$ & $13.9 \pm 1.1^{* *}$ \\
\hline
\end{tabular}

Note: *: represent that compared with model group $P<0.05$;**: represent that compared with model group $P<0.01$.

As Table 3 shows that compared with blank group, spleen index, thymus index level in model group has improved significantly $(P<0.01)$, it manifests inducing immunosuppressive model is successful . Compared with model group, thymus index in Lentinus Edodes Polysaccharide group , high-dose , mid-dose and low-dose jinjiang groups improved significantly on average $(P<0.01)$ and among them, high-dose jinjiang group is optimal . Compared with model group, spleen index in Lentinus Edodes Polysaccharide group, high-dose , mid-dose jinjiang groups improved significantly on average $(P<0.01)$ and among them, Lentinus Edodes Polysaccharide group is optimal. Spleen index in low-dose jinjiang group improved obviously on average $(P<0.05)$. 


\section{Effects on the transformation levels of peripheral blood lymphocytes and NK cells activity of immunosuppressive mice model induced by cyclophosphamide.}

Table4. Effects on the transformation levels of peripheral blood lymphocytes and NK cells activity of immunosuppressive mice model induced by cyclophosphamide $\left(\underline{\bar{x}}_{ \pm s}\right)$

\begin{tabular}{lllll}
\hline group & $\mathrm{n}$ & $\begin{array}{l}\text { Dose } \\
(\mathrm{g} / \mathrm{kg})\end{array}$ & $\begin{array}{l}\text { transformation } \\
\text { lymphocytes }(\%)\end{array}$ & $\begin{array}{l}\text { of } \\
\text { ity }\end{array}$ \\
\hline Blank group & 10 & - & $41.7 \pm 2.8^{* *}$ & $56.8 \pm 1.7^{* *}$ \\
Model group & 10 & - & $34.3 \pm 2.6$ & $47.1 \pm 2.3$ \\
Lentinus Edodes Polysaccharide group & 10 & 0.03 & $42.5 \pm 2.8^{* *}$ & $55.5 \pm 1.4^{* *}$ \\
high-dose jinjiang groups & 10 & 12 & $40.5 \pm 1.4^{* *}$ & $55.5 \pm 1.6^{* *}$ \\
mid-dose jinjiang groups & 10 & 6 & $38.9 \pm 2.6^{* *}$ & $52.8 \pm 1.9^{* *}$ \\
low-dose jinjiang groups & 10 & 3 & $37.9 \pm 2.7^{* *}$ & $48.7 \pm 1.5^{* *}$ \\
\hline
\end{tabular}

Note: **: represent that compared with model group $P<0.01$.

As Table 4 shows that compared with blank group, transformation of lymphocyteslevel, NK cells activity level in model group has improved significantly $(P<0.01)$, it manifests inducing immunosuppressive model is successful . Compared with model group , transformation of lymphocytes level , NK cells activity levelin Lentinus Edodes Polysaccharide group, high-dose , mid-dose and low-dose jinjiang groups improved significantly on average $(P<0.01)$ and among them, Lentinus Edodes Polysaccharide group is optimal.

\section{Discussion}

Traditional Chinese medicine holds that growth and decline between vital energy and pathogenic factor decides occurrence, development, prognosis of disease to raise to promote the body resistance and eliminate pathogenic factors as main treatment principle[2].

In the nature world, vital energy and pathogenic factor struggle continuously, which appears as alternative chills and fever. The parlance of alternative chills and fever stems from Canon of Medicine which is due to Qi deficiency and pathogenic factor. Qi deficiency and pathogenic factor exist in the progress of disease dialectically, objectively. Alternative chills and fever is closely related to asthenia and sthenia changes of disease, so when we want to prevent disease and protect health, we must aviod body deficiency and pathogenic in the exterior, and conserve vital qi in the interior ; Treatment should emphasize to promote the body resistance and eliminate pathogenic factors, to eliminate pathogenic factors not to injure body resistance , strengthen body resistance not to conserve pathogenic factors[3]. Vital qi in body is all along to fight against pathogenic factors in the environment. In the induced pathogenic factor environment, some people fall ill, someone not . Some people fall ill with onset of symptoms immediately, some people not. When vital energy and pathogenic factor struggle, vital energy is dominant all the time and can defeat pathogenic factor. That is why it is very common that people are always healthy, and rare to be ill . The treatment principle is to build up health , improve the ability of resisting external pathogenic factor and antivirus $[4,5]$.

According to the principles of Traditional Chinese Medicine establishing principles based on differental diagnosis and selecting medicines principles, we select honeysuckle, gringer, taraxacum, coix seed, angelica, jujube, chrysanthemum indicum to compose prescription. The formula of Jinjiang granules is composed of the extract of honeysuckle, gringer, taraxacum, coix seed, angelica, jujube, chrysanthemum indicum and lactose. Jinjiang granules have the function of heat- clearing and detoxifying, dispelling wind-heat, relieving sore throat, preventing from cold, improving immuni- 
ty. Honeysuckle in the prescription is cold in property, with favourite odour, clearing heat but not to injure stomach, fragrant and to goad pathogenic factor. It can not only dispel wind and heat, but also detoxify, relieve sore throat, remove summer-heat and apprehension; Taraxacum can clear heat and detoxify, detumescence; Chrysanthemum indicum can clear heat and detoxify, disperse wind and pacify liver. When these three medicine were used together, the ability of heat-clearing and detoxifying is doubling. Ginger can induce diaphoresis, warm middle energizer to arrest vomiting, relieve coughs; Angelica can get rid of colds, relieve stuffy nose and pain. When these two medicine were used together, it can enhance the ability of relieving the exterior and dispelling pathogenic factor. Coix seed invigorate the spleen and excrete dampness, antidiarrheal. Jujube replenish qi and blood, tranquilize. When these two medicine were used together, it can strengthen vital energy, dispel pathogenic factor, and prevent pathogenic factor invade body again. The whole prescription includes warm and cold, invigoration and purgation . Clearing intestinal contents should be primary, while strengthening body resistance should be complementary. They can dispel the pathological factor from the exterior of the body, clear heat and detoxify in order to attack heat-toxin pathogenic factor, strengthen vital energy, build up health, and enhance the ability of disease resistance. It can be applied to the people whose immunity is lower or who is inclined to be cold to improve body`s immunity .

Immune cells is the cell which can mediate or participate in immune response[6].Among them, monocytes - phagocytes systerm is important immune cell in vivo . It not only plays animportant part in immune response, but also can clean up aging, damaged cells and some decay substance to retain stability of internal environment in living creature . In addition, Immuneglobulin (Ig) is immune molecules that plays a role in immune response, and humoral immune function in vivo is related closely to the level of immuneglobulin. When specific antigen is combined with immuneglobulin, immuneglobulin can generate two action of immune defense. On the one han it can take a direct effect such as neutralizing bacterial toxin and preventing the combination with other cells etc. . On the other hand, it can take a indirect effect, such as killing antigen with the participation of complement system, NK cells or phagocytes .

Spleen is the bigest immune organs of body with a lot of lymphocytes and it is the hub of cellular immunity and humoral immunity . Lymphocytes is immunocompetent cell of body, and an important part of acquired immunity. Immune function of body can be reflected indirectly by the proliferation ability, for example lymphocytes activate and differentiate into immunocompetent cells to enhance the ability of immune response $[7,8]$.

NK cells is the first defense line in the innate immunity. It plays a role in immune surveillance and is the key cells of acquired immunoregulation [9] . Detecting killing ability of NK cells can help us to know the functional status of NK cells and the importance, mechanism of it under pathological, physiological state [10] .

Cyclophosphamide is as a immunosuppressant . Lots of research shows that cyclophosphamide as a nitrogen mustard alkylating agent can reduce the quantity of lymphocytes to inhibate humoral and cellular immune response and induce animals to be immunosuppressive[11], so the mice were injected intraperitoneally cyclophosphamide to make immunosuppressive models in the experiment . Lentinus Edodes Polysaccharide can impove body`s immunity, that`s why we select it as a positive control drug.

The research takes murineorgan index test, hemolysin and hemolytic plaque test ,transformation of peripheral blood lymphocytes test, the ability of peritoneal ma- 
crophage phagocytosing chicken red blood cells test, detecting activity of spleen tissueNK cell test with immunosuppressive mice model .

Compared with blank group, there are significant differences in indexes of immunosuppressive mice model .Compared with immunosuppressivemodel group , murineorgan index, the level of hemolysin and hemolytic plaque, transformation ability of peripheral blood lymphocytes, the ability of peritoneal macrophage phagocytosing chicken red blood cells, the activity of NK cell would be enhanced in medication group. It suggests that jinjiang granules can improve the immunity of immunosuppressive mice and the high-dosejinjiang group is optimal among them .

\section{References}

[1] Ming-san Miao, Ruiqi Li, Xue-xia Zhang. Effect of Jin Jiang Granules on Immune Function in NormalMice. Advanced Materials Research, 850-851(2014):1259-1262.

[2] Mu-chuan Zhang, Zhang Qi-cheng. Features of HU Xi-shu's cognitive pattern of'Following the Flow'based on the conception of Zheng Xie Jiao Zheng'. CJTCMP. 04(2012):874-878.

[3] Zhuo Song, Chun-yu Su, Jing-nan Xu, Jie Li. Theoretical Basis and Evidence-based Basis of Supporting Healthy Energy and Removing Toxicity Method in Preventing and Treating Stomach Cancer. Journal of Traditional Chinese Medicine, 10(2016):829-833.

[4] Dong-hui Yue, Yan Bi, Yan-Song, et al. Research on TCM treatment of influenza . CJTCMP,12(2015):4404-4407.

[5] HaoWu, Qin-you Ren. Progress of Traditional Chinese Medicine Treatment of Malignant Tumor. Liangning Journal of Traditional Chinese Medicine , 04, (2016):876-878.

[6] Guang-wei Liu. Innate immune defense effects of pathogenic microorganism infection. Chinese Science Bulletin,27,(2016):2986-2995.

[7] Ying-fu Liu, Yue Fu, Lu Hao, et al. The influence of resveratrol on immunoregulatory activity ofmouse splenic lymphocyte. Science and Technology of Food Industry, 08(2016):335-339.

[8] Gao-lin Zhang, Wei-dongCheng, Wen-jun Zhang, et al. Effects of serums containing Buzhong Yiqi decoction with Astragalus Radix or Hedysari Radix on anti-immunosenescence in spleen lymphocytes of SAMP8 mice. China Journal of Chinese Materia Medica,15(2016):2888-2894.

[9] Zhi-gang Tian, Yong-yan Chen. Development, differentiation and immune recognition of natural killer cells . Journal of Chinese Immunology,01(2009):31-34.

[10] Jin Zhou, Hui Peng, Zhi-gang Tian .Research progress on negative regulation of adaptive immune response by NK cells. Journal of Chinese Immunology, 06(2016):769-776.

[11] WeiCui, SaLiu, MinYang, et al. Study on inhibitory effect of combined administration of bear bile powder and cyclophosphamide on colorectal cancer liver metastasis byregulating tumor microenvironment. China Journal of Chinese Materia Medica, 07(2013):1036-1040. 